NT@UW-019

MIT-CTP 3405

\title{
Soft Pion Emission in DVCS
}

\author{
Jiunn-Wei Chen ${ }^{a, b}$, and Martin J. Savage ${ }^{c}$ \\ a Department of Physics, National Taiwan University, \\ Taipei 10617, Taiwan. \\ ${ }^{b}$ Center for Theoretical Physics, Massachusetts Institute of Technology, \\ Cambridge, MA 02139. \\ ${ }^{c}$ Department of Physics, University of Washington, \\ Seattle, WA 98195.
}

\begin{abstract}
We examine soft-pion emission in deeply virtual Compton scattering. Contrary to previous claims, we find that the amplitude for soft-pion emission is not directly related to the generalized parton distributions in the nucleon.
\end{abstract}

August 2003 


\section{INTRODUCTION}

Deep inelastic scattering (DIS) from the nucleon, $e N \rightarrow e^{\prime} X$, has played a central role in the development of our understanding of QCD. Application of the optical theorem to the inclusive cross-section for DIS relates it to the forward matrix element of the time-ordered product of two electromagnetic currents. As QCD is asymptotically free at short-distances, an operator product expansion of the time-ordered product can be performed which allows the total inclusive cross section to be related to the forward matrix elements in the nucleon of twist-2 operators involving the quark and gluon fields. One particularly nice implication of this is that these forward matrix elements correspond to the moments of the parton distribution functions (PDF's) in the nucleon, where the PDF's are probability distributions. Recently, it has been realized that the off-forward matrix elements of these twist-2 operators are also of interest [1-8]. Of particular note is the relation between the off-forward matrix elements of the quark and gluon energy-momentum tensors and the fraction of the total angular momentum of the nucleon carried by the quarks and gluons [1,2]. Away from the forward direction the idea of a generalized parton distribution (GPD) has been introduced to provide a somewhat unified description of the scattering processes [1-8]. While the forward limit of the GPD's reproduce the PDF's, weighted integration of the GPD's over Bjorken $x$ reproduce the form-factors of the twist-2 operators in the nucleon (for a nice review see Filippone and Ji [9]). Gravitational scattering is not a feasible option for the measurement of the matrix element of the energy-momentum tensor, however, deeply virtual Compton scattering (DVCS) [2,10-12] provides an experimentally realized alternate process with which to explore the matrix elements of the twist-2 operators over a wide range of kinematics.

A significant experimental effort has been mounted to measure the GPD's through DVCS [13-16]. In general, each GPD has three kinematic arguments, $x, \xi$ and $t$, e.g. $H^{q}(x, \xi, t)$. Elastic DVCS with high energy electrons is determined by a convolution in the variable $x$ of the GPD's with $1 /( \pm x-\xi+i \epsilon)$, and therefore one cannot determine the GPD's directly from such measurements. However, at lower energies and with polarized beams one can determine the imaginary part of this convolution which gives the GPD's evaluated at $x=\xi$, e.g. Ref. [15]. Soft-pion emission during DVCS $\gamma^{*} p \rightarrow \gamma p \pi$ may provide a background to the elastic process $\gamma^{*} p \rightarrow \gamma p$ due to limited detector resolution in some experiments. The matrix elements and cross section for soft-pion emission during DVCS (DVCS-pion production) have been computed by Guichon, Mosse and Vanderhaeghen [17] using current algebra. They find that there are no further unknown strong interaction contributions beyond the GPD's of the nucleon. In this paper we compute the amplitude for soft-pion emission during DVCS (DVCS-pion production) using chiral perturbation theory. We find that at leading order in the chiral expansion, the amplitude for DVCS-pion production is controlled by the PDF's and by additional strong interaction parameters that are not related to the elastic GPD's. This result disagrees with the result of Ref. [17].

\section{TWIST-2 MATRIX ELEMENTS AND CHIRAL PERTURBATION THEORY}

The leading order chiral Lagrangian describing the low-energy dynamics of the nucleons, $\Delta$ 's and pions (pseudo-Goldstone bosons) that is consistent with the spontaneously broken $S U(2)_{L} \otimes S U(2)_{R}$ approximate chiral symmetry of QCD is $[18,19]$ 


$$
\begin{aligned}
\mathcal{L}= & \bar{N} i v \cdot \mathcal{D} N-\bar{T}_{\mu} i v \cdot \mathcal{D} T^{\mu}+\Delta \bar{T}_{\mu} T^{\mu} \\
& +\frac{f^{2}}{8} \operatorname{Tr}\left[\partial_{\mu} \Sigma^{\dagger} \partial^{\mu} \Sigma\right]+\lambda \frac{f^{2}}{4} \operatorname{Tr}\left[m_{q} \Sigma^{\dagger}+\text { h.c. }\right] \\
& +2 g_{A} \bar{N} S^{\mu} A_{\mu} N+g_{\Delta N}\left[\bar{T}^{a b c, \nu} A_{a, \nu}^{d} N_{b} \epsilon_{c d}+\text { h.c. }\right]+2 g_{\Delta \Delta} \bar{T}_{\nu} S^{\mu} A_{\mu} T^{\nu}
\end{aligned}
$$

where $\mathcal{D}$ is the chiral covariant derivative, $N$ is the nucleon field operator, and $T^{\mu}$ is the Rarita-Schwinger field containing the quartet of spin- $\frac{3}{2} \Delta$-resonances. The mass difference between the $\Delta$-resonances and the nucleon is $\Delta$, and the axial couplings between the baryons and pions are $g_{A}, g_{\Delta N}$ and $g_{\Delta \Delta} . S_{\mu}$ is the covariant spin vector defined in heavy-baryon $\chi \mathrm{PT}[18,19]$, and $v_{\mu}$ is the heavy-baryon four-vector, with $v^{2}=1$. The pions appear through $\Sigma$ and $A_{\mu}$ which are defined to be

$$
\Sigma=\exp \left(\frac{2 i M}{f}\right)=\xi^{2}, A^{\mu}=\frac{i}{2}\left(\xi \partial^{\mu} \xi^{\dagger}-\xi^{\dagger} \partial^{\mu} \xi\right), M=\left(\begin{array}{cc}
\pi^{0} / \sqrt{2} & \pi^{+} \\
\pi^{-} & -\pi^{0} / \sqrt{2}
\end{array}\right) .
$$

We have not shown flavor indices in eq. (1) for terms where the contractions are unambiguous. Under chiral transformations the various fields transform as

$$
\Sigma \rightarrow L \Sigma R^{\dagger}, \quad N \rightarrow U N \quad, \quad T^{\mu} \rightarrow U U U T^{\mu} \quad, \quad A^{\mu} \rightarrow U A^{\mu} U^{\dagger}
$$

where $L$ and $R$ denote left- and right-handed chiral transformations respectively. The $\xi$ field (not to be confused with the kinematic variable $\xi$ that appears as an argument of the GPD's) transforms as $L \xi U^{\dagger}=U \xi R^{\dagger}$, which defines the transformation $U$. The mass-matrix, which is treated as a spurion field, is taken to transform as $m_{q} \rightarrow L m_{q} R^{\dagger}$.

The twist-2 operators involving the quark fields that provide the dominant contribution to DIS and DVCS are

$$
\begin{aligned}
& \theta_{V, \mu_{1} \ldots \mu_{n}}^{(n), 0}=(i)^{n-1} \bar{q} \gamma_{\left\{\mu_{1}\right.} \stackrel{\leftrightarrow}{D}_{\mu_{2}} \ldots \stackrel{\leftrightarrow}{D}_{\left.\mu_{n}\right\}} q \\
& \theta_{V, \mu_{1} . \mu_{n}}^{(n), b}=(i)^{n-1} \bar{q} \gamma_{\left\{\mu_{1}\right.} \stackrel{\leftrightarrow}{D}_{\mu_{2}} \ldots \stackrel{\leftrightarrow}{D}_{\left.\mu_{n}\right\}} \tau^{b} q \\
& \theta_{A, \mu_{1} . \mu_{n}}^{(n), 0}=(i)^{n-1} \bar{q} \gamma_{\left\{\mu_{1}\right.} \stackrel{\leftrightarrow}{D}_{\mu_{2}} \ldots \stackrel{\leftrightarrow}{D}_{\left.\mu_{n}\right\}} \gamma_{5} q \\
& \theta_{A, \mu_{1} . \mu_{n}}^{(n), b}=(i)^{n-1} \bar{q} \gamma_{\left\{\mu_{1}\right.} \stackrel{\leftrightarrow}{D}_{\mu_{2}} \ldots \stackrel{\leftrightarrow}{D}_{\left.\mu_{n}\right\}} \gamma_{5} \tau^{b} q
\end{aligned}
$$

where the indices enclosed by $\{\ldots\}$ are symmetrized and their traces are removed. The operator $\stackrel{\leftrightarrow}{D}_{\mu}$ is $\stackrel{\leftrightarrow}{D}_{\mu}=\vec{D}_{\mu}-\overleftarrow{D}_{\mu}$, where $D_{\mu}=\partial_{\mu}+i g A_{\mu}$ is the QCD covariant derivative, and $g$ is the strong coupling constant. Hadronic matrix elements of the operators in eq. (4) in the kinematic regimes appropriate for DIS and slightly off-forward DVCS can be described in chiral perturbation theory $(\chi \mathrm{PT})[20-25]$ as the momentum transfer to the hadronic system is small compared to the scale of chiral symmetry breaking, $\Lambda_{\chi} \sim 1 \mathrm{GeV}$.

Matrix elements of the isoscalar operators $\theta_{V, \mu_{1} . \mu_{n}}^{(n), 0}$ and $\theta_{A, \mu_{1} \ldots \mu_{n}}^{(n), 0}$ in the nucleon are reproduced at leading order in the chiral expansion by the operators [20,21]

$$
\begin{aligned}
& \theta_{V, \mu_{1} . \mu_{n}}^{(n), 0} \rightarrow M^{n-1}\left\langle x^{n-1}\right\rangle_{q_{V}^{(0)}} v_{\mu_{1}} v_{\mu_{2}} \ldots v_{\mu_{n}} \bar{N} N \\
& \theta_{A, \mu_{1} . \mu_{n}}^{(n), 0} \rightarrow M^{n-1}\left\langle x^{n-1}\right\rangle_{q_{A}^{(0)}} \quad v_{\left\{\mu_{1}\right.} v_{\mu_{2}} \ldots v_{\mu_{n-1}} \bar{N} S_{\left.\mu_{n}\right\}} N \text {, }
\end{aligned}
$$


where $\left\langle x^{p}\right\rangle_{q_{V}^{(0)}}$ and $\left\langle x^{p}\right\rangle_{q_{A}^{(0)}}$ are the $p^{\prime}$ th moments of the isoscalar vector and axial-vector PDF's in the nucleon, respectively. $M$ is the nucleon mass.

To construct matrix elements of the isovector operators one introduces the spurion fields $\tau_{L}^{a}$ and $\tau_{R}^{a}$ which transform as

$$
\tau_{L}^{a} \rightarrow L \tau_{L}^{a} L^{\dagger}, \quad \tau_{R}^{a} \rightarrow R \tau_{R}^{a} R^{\dagger}
$$

under $S U(2)_{L} \otimes S U(2)_{R}$ and whose vevs are $\left\langle\tau_{L}^{a}\right\rangle=\left\langle\tau_{R}^{a}\right\rangle=\tau^{a}$. It is then convenient to define

$$
\tau_{\xi, \pm}^{b}=\frac{1}{2}\left(\xi^{\dagger} \tau_{L}^{b} \xi \pm \xi \tau_{R}^{b} \xi^{\dagger}\right)
$$

that transforms as $\tau_{\xi, \pm}^{b} \rightarrow U \tau_{\xi, \pm}^{b} U^{\dagger}$ under $S U(2)_{L} \otimes S U(2)_{R}$, and as $\tau_{\xi, \pm}^{b} \rightarrow \pm \tau_{\xi, \pm}^{b}$ under parity. At leading order in the chiral expansion the matrix elements of the isovector operators $\theta_{V, \mu_{1} . \mu_{n}}^{(n), b}$ and $\theta_{A, \mu_{1} . . \mu_{n}}^{(n), b}$ in the nucleon and between the nucleon and $\Delta$ (required for our calculation of soft-pion emission) are reproduced by

$$
\begin{aligned}
& \theta_{V, \mu_{1} . . \mu_{n}}^{(n), b} \rightarrow M^{n-1}\left\langle x^{n-1}\right\rangle_{q_{V}^{(1)}} v_{\mu_{1}} v_{\mu_{2}} \ldots v_{\mu_{n}} \bar{N} \tau_{\xi,+}^{b} N \\
& +M^{n-1} a^{(n)} v_{\left\{\mu_{1}\right.} v_{\mu_{2}} \ldots v_{\mu_{n-1}} \bar{N} S_{\left.\mu_{n}\right\}} \tau_{\xi,-}^{b} N \\
& \theta_{A, \mu_{1} \ldots \mu_{n}}^{(n), b} \rightarrow M^{n-1}\left\langle x^{n-1}\right\rangle_{q_{A}^{(1)}} v_{\left\{\mu_{1}\right.} v_{\mu_{2}} \ldots v_{\mu_{n-1}} \bar{N} S_{\left.\mu_{n}\right\}} \tau_{\xi,+}^{b} N \\
& +M^{n-1} b^{(n)} v_{\mu_{1}} v_{\mu_{2}} \ldots v_{\mu_{n}} \bar{N} \tau_{\xi,-}^{b} N \\
& +M^{n-1} c^{(n)} v_{\left\{\mu_{1}\right.} v_{\mu_{2}} \ldots v_{\mu_{n-1}}\left[\bar{T}_{\left.\mu_{n}\right\}}^{i j k}\left(\tau_{\xi,+}^{b}\right)_{i}^{l} N_{j} \epsilon_{k l}+\text { h.c. }\right] \text {, }
\end{aligned}
$$

where $\left\langle x^{p}\right\rangle_{q_{V}^{(1)}}$ and $\left\langle x^{p}\right\rangle_{q_{A}^{(1)}}$ are the $p$ 'th moments of the isovector vector and axial-vector PDF's in the nucleon, respectively. The coefficients $a^{(n)}, b^{(n)}$, and $c^{(n)}$ cannot be related to the PDF's in the nucleon (and hence the GPD's) by chiral symmetry. This disagrees with the conclusion of Guichon, Mosse and Vanderhaeghen [17] who claim that chiral symmetry alone is sufficient to relate $a^{(n)}$ and $b^{(n)}$ to $\left\langle x^{n-1}\right\rangle_{q_{V}^{(1)}}$ and $\left\langle x^{n-1}\right\rangle_{q_{A}^{(1)}}$ in the kinematic regime $m_{\pi}^{2} \ll-t \ll \Lambda_{\chi}^{2}$.

At higher orders in the chiral expansion there will be contributions from local operators that depend upon $v \cdot q / \Lambda_{\chi}$ (the energy transfer to the hadrons in their rest frame) and $q^{2} / \Lambda_{\chi}^{2}$ (where $q^{2}=t$ ). These operators, along with pion loop contributions, will contribute to the off forward-matrix elements and will provide the low-energy behavior of the GPD's. Further, there will be additional operators whose matrix elements between single nucleon states vanish, but can contribute to DVCS-pion production.

We have not shown the matrix elements of the twist-2 operators in the pion sector [20]. For an operator with $n$ indices, the matrix element between single pion states is suppressed by factors of $p_{\pi}^{n}$, where $p_{\pi}$ is the pion four-momentum. For $n \geq 2$ the contribution to DVCS-pion production from insertions of the twist-2 operators into the pion is a higher order contribution.

\section{DVCS-PION PRODUCTION OFF THE NUCLEON}

It is straightforward to compute the leading order contribution to $\gamma^{*} N \rightarrow \gamma N \pi$ in $\chi \mathrm{PT}$. Leading order corresponds to $\mathcal{P}^{0}$ in the power-counting, where $\mathcal{P} \sim k, q, \Delta, m_{\pi}$ are the small 
expansion parameters, where $k$ is the pion four-momentum and $q$ is the four-momentum injected by the twist- 2 operator. We compute just one of the possible processes, $\gamma^{*} p \rightarrow$ $\gamma n \pi^{+}$, in this work and the matrix elements for other possible initial and final states can be constructed analogously. At leading order in the chiral expansion, the matrix elements for
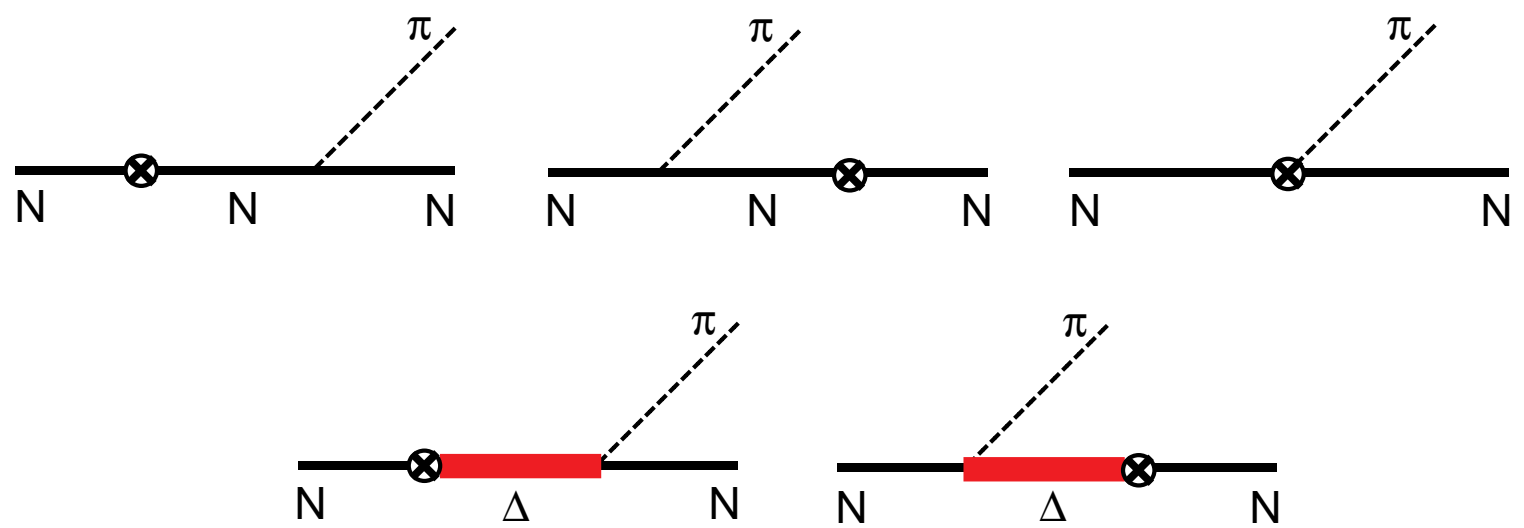

FIG. 1. The tree-level diagrams that give the leading order contributions to DVCS-pion production for $n \geq 2$. The crossed-circle denotes an insertion of a twist-2 operator.

$n \geq 2$ result from the diagrams shown in Fig. 1, and are found to be

$$
\begin{aligned}
& \left\langle n \pi^{+}\left|\theta_{V, \mu_{1} . \mu_{n}}^{(n), 0}\right| p\right\rangle=0 \\
& \left\langle n \pi^{+}\left|\theta_{V, \mu_{1} . . \mu_{n}}^{(n), 3}\right| p\right\rangle=-i \frac{4 g_{A}}{f} M^{n-1}\left\langle x^{n-1}\right\rangle_{q_{V}^{(1)}} \frac{v_{\mu_{1}} v_{\mu_{2}} \ldots v_{\mu_{n}}}{v \cdot q} \bar{U}_{n} S \cdot k U_{p} \\
& -i M^{n-1} \frac{2 a^{(n)}}{f} v_{\left\{\mu_{1}\right.} v_{\mu_{2}} \ldots v_{\mu_{n-1}} \bar{U}_{n} S_{\left.\mu_{n}\right\}} U_{p} \\
& \left\langle n \pi^{+}\left|\theta_{A, \mu_{1} . \mu_{n}}^{(n), 0}\right| p\right\rangle=i \frac{2 g_{A}}{f} M^{n-1}\left\langle x^{n-1}\right\rangle_{q_{A}^{(0)}} \frac{v_{\left\{\mu_{1}\right.} v_{\mu_{2}} \ldots v_{\mu_{n-1}}}{v \cdot q} i \epsilon^{\mu_{n\}} \alpha \lambda \sigma} k_{\alpha} v_{\lambda} \bar{U}_{n} S_{\sigma} U_{p} \\
& \left\langle n \pi^{+}\left|\theta_{A, \mu_{1} . \mu_{n}}^{(n), 3}\right| p\right\rangle=-i \frac{g_{A}}{f} M^{n-1}\left\langle x^{n-1}\right\rangle_{q_{A}^{(1)}} \frac{v_{\left\{\mu_{1}\right.} v_{\mu_{2}} \cdots v_{\mu_{n-1}}}{v \cdot q}\left(v \cdot q v_{\left.\mu_{n}\right\}}-k_{\left.\mu_{n}\right\}}\right) \bar{U}_{n} U_{p} \\
& -i M^{n-1} \frac{2 b^{(n)}}{f} v_{\mu_{1}} v_{\mu_{2}} \ldots v_{\mu_{n}} \bar{U}_{n} U_{p} \\
& +i M^{n-1} \frac{8}{9} \frac{g_{\Delta N} c^{(n)}}{f} \frac{v_{\left\{\mu_{1}\right.} v_{\mu_{2}} \ldots v_{\mu_{n-1}}}{(v \cdot q)^{2}-\Delta^{2}} \bar{U}_{n}\left[v \cdot q\left(v \cdot q v_{\left.\mu_{n}\right\}}-k_{\left.\mu_{n}\right\}}\right)\right. \\
& \left.+i \Delta \varepsilon_{\left.\mu_{n}\right\} \alpha \sigma \lambda} k^{\alpha} v^{\sigma} S^{\lambda}\right] U_{p},
\end{aligned}
$$

where we have used $v \cdot q=v \cdot k$ in the heavy baryon limit, and where it is clear that all contributions to these matrix elements are $\mathcal{O}\left(\mathcal{P}^{0}\right)$ in the power-counting. $U_{p}$ and $U_{n}$ are the spinors associated with the proton and neutron respectively. The matrix elements will be modified near the peak of the $\Delta$-pole, where the $\Delta$-width will need to be resummed into the propagator. It is important to note that the matrix elements in eq. (9) are the leading order contribution for all kinematics such that $(v \cdot q)^{2},|t|, m_{\pi}^{2} \ll \Lambda_{\chi}^{2}$ for any value of the ratio $t / m_{\pi}^{2}$. We do not go on to compute the differential and total cross sections, as this can 
be done straightforwardly and does not add anything to our discussion. The point is that there are additional contributions to the DVCS-pion production cross section that are not related to the nucleon PDF's (GPD's), as encapsulated by the constants $a^{(n)}$ and $b^{(n)}$. A similar feature is probably true for any generic DVCS inelastic process, where the PDF's or GPD's of the nucleon can only describe part of the contribution.

\section{CURRENT CONSERVATION AND RELATIONS BETWEEN COEFFICIENTS}

Only the chiral transformation properties of the twist-2 operators were used in the construction of the most general set of leading order operators in eq. (5) and eq. (8). As such, we did not include the fact that the $n=1$ vector operators are conserved and the $n=1$ axial operators are partially conserved. These conservation laws lead to additional constraints on the coefficients $a^{(1)}$ and $b^{(1)}$. We have only presented the matrix elements for $\gamma^{*} p \rightarrow \gamma n \pi^{+}$

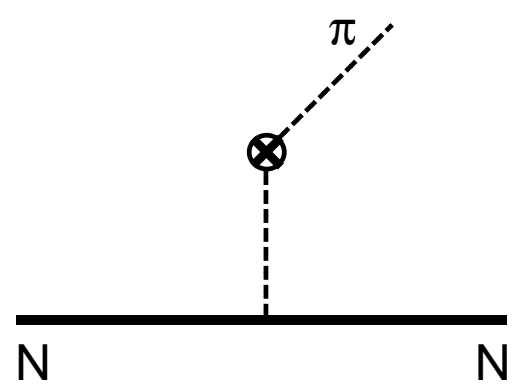

FIG. 2. The additional tree-level diagram that contributes at leading order to the $n=1$ matrix element for DVCS-pion production of the isovector-vector twist-2 operator (the isovector-vector current operator). The crossed-circle denotes an insertion of the $n=1$ twist-2 operator.

for $n \geq 2$ in eq. (9) as the $n=1$ matrix elements are trivial. However, it is useful to explore the impact of current conservation on the $n=1$ vector-isovector matrix element, which receives an additional contribution at leading order from the diagram in Fig. 2, and is found to be

$$
\begin{aligned}
\left\langle n \pi^{+}\left|\theta_{V, \mu}^{(1), 3}\right| p\right\rangle= & -i \frac{4 g_{A}}{f} \frac{v_{\mu}}{v \cdot q} \bar{U}_{n} S \cdot k U_{p}-i \frac{2 a^{(1)}}{f} \bar{U}_{n} S_{\mu} U_{p} \\
& -i \frac{4 g_{A}}{f} \bar{U}_{n} \frac{S \cdot(k-q)(2 k-q)^{\mu}}{(k-q)^{2}-m_{\pi}^{2}} U_{p} .
\end{aligned}
$$

Conservation of the vector current $\bar{q} \gamma_{\mu} \tau^{a} q$ requires that $q^{\mu}\left\langle n \pi^{+}\left|\theta_{V, \mu}^{(1), 3}\right| p\right\rangle=0$, and hence

$$
q^{\mu}\left\langle n \pi^{+}\left|\theta_{V, \mu}^{(1), 3}\right| p\right\rangle=-i \frac{2}{f}\left[a^{(1)}+2 g_{A}\right] \bar{U}_{n} S \cdot q U_{p}=0
$$

from which we conclude that $a^{(1)}=-2 g_{A}$. This agrees with the known result derived directly from the vector current operator in heavy-baryon $\chi \mathrm{PT}[18,19]$ and is the relation 
that was found in Ref. [17]. In addition, Ref. [17] makes the more general statement that $a^{(n)}=-2\left\langle x^{n-1}\right\rangle_{q_{A}^{(1)}}$, which reproduces the $n=1$ result as $\langle 1\rangle_{q_{A}^{(1)}}=g_{A}$.

Lets consider the implication of having $a^{(n)}=-2\left\langle x^{n-1}\right\rangle_{q_{A}^{(1)}}$, as claimed in Ref. [17]. Specifically, consider the $n=2$ isovector operator $\theta_{V, \mu \nu}^{(2), b}$, which is not conserved due to interactions with the gluon field, and it is straightforward to show that in the chiral limit

$$
\partial_{\mu} \theta_{V, \mu \nu}^{(2), 3}=-4 g \bar{q} \gamma^{\alpha} G_{\alpha \nu} \tau^{3} q
$$

where $G_{\alpha \beta}$ is the gluon field strength tensor, defined by $\left[D_{\alpha}, D_{\beta}\right]=i g G_{\alpha \beta}$, and we have used the equation of motion $\not D q=0$. In the effective theory, one finds that

$$
\begin{aligned}
-i q^{\mu}\left\langle n \pi^{+}\left|\theta_{V, \mu \nu}^{(2), 3}\right| p\right\rangle= & -\frac{4 g_{A}}{f} M\langle x\rangle_{q_{V}^{(1)}} v_{\nu} \bar{U}_{n} S \cdot k U_{p} \\
& -M \frac{a^{(2)}}{f} \bar{U}_{n}\left(v \cdot q S_{\nu}+v_{\nu} S \cdot q\right) U_{p} .
\end{aligned}
$$

Given that one cannot presently compute the matrix element of $\partial_{\mu} \theta_{V, \mu \nu}^{(2), 3}$, and chiral symmetry alone does not relate $a^{(n)}$ to single nucleon matrix elements, we conclude that the assertion of Ref. [17] is false.

There are a few assertions in Ref. [17] that have contributed in some part to their error. First, the non-local operator that arises at the scale of the scattering, $Q^{2}$, was used in all operator manipulations. The difficulties in dealing with non-local operators are well-known, and efforts to systematize non-local effective field theory have shown it to be significantly more complex [26] than local effective field theories. A straightforward problem with this can be seen in the fact that $\theta_{V, \nu}^{(1), 3}$ and $\theta_{V, \mu_{1} . . \mu_{10}}^{(10), 3}$ originate from the same non-local operator, but their scale dependence due to strong-interactions are quite different. Second, after rederiving pole-ology (something that has been well understood for decades) a central feature of their soft-pion analysis requires them to work at non-zero quark mass, and they are unable to derive their result working exclusively in the chiral limit. Hand waving arguments, including the unjustifiable neglect of $\left[\frac{d}{d t} Q_{5}, \theta_{V, A}^{(n)}\right]$, are required in order for them to consider the chiral limit. We believe that these two aspects of the calculation of Ref. [17] are flawed. During the thirty or more years since the techniques used in Ref. [17] were introduced, significant progress has been made in understanding how to deal with the approximate chiral symmetry of QCD. We have used these well-known modern tools in our analysis.

\section{CONCLUSIONS}

We have explored soft-pion production associated with DVCS. Using the transformation properties of the twist-2 operators under the $S U(2) \otimes S U(2)$ chiral symmetry we have shown that there are contributions to DVCS-pion production that are not related to the GPD's of the nucleon, even at leading order in the chiral expansion. This contradicts recent claim in Ref. [17]. Further, the contribution from nucleon structure depends upon the PDF's and one does not need to consider the GPD's at leading order. Therefore, we conclude that DVCS-pion production does not depend only on the GPD's of the nucleon, and gives rise to a presently incalculable background to extracting GPD's from DVCS in some experiments. 
However, naive dimensional analysis can be used to make a rough estimate of the unknown coefficients, and hence a rough estimate of the size of the background. It may be the case that a combined study of one-pion and two-pion emission during DVCS will allow for a determination of the constants $a^{(n)}, b^{(n)}$ and $c^{(n)}$. However, there are additional contributions to this process coming from operators that contribute to $\gamma^{*} N \rightarrow \gamma \Delta \pi$ but not to $\gamma^{*} N \rightarrow \gamma \Delta$. A significantly more comprehensive investigation is required in order to ascertain if it is possible to disentangle all contributions at leading order in the chiral expansion.

\section{ACKNOWLEDGMENTS}

We would like to thank Xiang-Dong Ji, David Kaplan and Jerry Miller for useful discussions. JWC thanks the Institute of Nuclear Theory at the University of Washington for its

hospitality. JWC is supported, in part, by the National Science Council of ROC and the U.S. department of energy under grant DOE/ER/40762-213. MJS is supported in part by the U.S. Dept. of Energy under Grant No. DE-FG03-97ER4014. 


\section{REFERENCES}

[1] X. D. Ji, Phys. Rev. Lett. 78, 610 (1997) [arXiv:hep-ph/9603249].

[2] X. D. Ji, Phys. Rev. D 55, 7114 (1997) [arXiv:hep-ph/9609381].

[3] F. M. Dittes, D. Muller, D. Robaschik, B. Geyer and J. Horejsi, Phys. Lett. B 209, 325 (1988).

[4] D. Muller, D. Robaschik, B. Geyer, F. M. Dittes and J. Horejsi, Fortsch. Phys. 42, 101 (1994) [arXiv:hep-ph/9812448].

[5] J. C. Collins, L. Frankfurt and M. Strikman, Phys. Rev. D 56, 2982 (1997) [arXiv:hep$\mathrm{ph} / 9611433]$.

[6] A. V. Radyushkin, Phys. Lett. B 385, 333 (1996) [arXiv:hep-ph/9605431].

[7] A. V. Radyushkin, Phys. Rev. D 56, 5524 (1997) [arXiv:hep-ph/9704207].

[8] A. V. Radyushkin, Phys. Rev. D 59, 014030 (1999) [arXiv:hep-ph/9805342].

[9] B. W. Filippone and X. D. Ji, Adv. Nucl. Phys. 26, 1 (2001) [arXiv:hep-ph/0101224].

[10] X. D. Ji and J. Osborne, Phys. Rev. D 58, 094018 (1998) [arXiv:hep-ph/9801260].

[11] A. V. Radyushkin, Phys. Lett. B 380, 417 (1996) [arXiv:hep-ph/9604317].

[12] J. C. Collins and A. Freund, Phys. Rev. D 59, 074009 (1999) [arXiv:hep-ph/9801262].

[13] A. Airapetian et al. [HERMES Collaboration], Phys. Rev. Lett. 87, 182001 (2001) [arXiv:hep-ex/0106068].

[14] S. Stepanyan et al. [CLAS Collaboration], Phys. Rev. Lett. 87, 182002 (2001) [arXiv:hep-ex/0107043].

[15] N. d'Hose, E. Burtin, P. A. Guichon, S. Kerhoas-Cavata, J. Marroncle and L. Mosse, Nucl. Phys. A 711, 160 (2002).

[16] L. Favart, Nucl. Phys. A 711, 165 (2002) [arXiv:hep-ex/0207030].

[17] P. A. Guichon, L. Mosse and M. Vanderhaeghen, arXiv:hep-ph/0305231.

[18] E. Jenkins and A. V. Manohar, Phys. Lett. B 255, 558 (1991).

[19] E. Jenkins and A. V. Manohar, UCSD-PTH-91-30 Talk presented at the Workshop on Effective Field Theories of the Standard Model, Dobogoko, Hungary, Aug 1991.

[20] D. Arndt and M. J. Savage, Nucl. Phys. A 697, 429 (2002) [arXiv:nucl-th/0105045].

[21] J. W. Chen and X. D. Ji, Phys. Lett. B 523, 107 (2001) [arXiv:hep-ph/0105197].

[22] J. W. Chen and X. D. Ji, Phys. Lett. B 523, 73 (2001) [arXiv:hep-ph/0105296].

[23] J. W. Chen and X. D. Ji, Phys. Rev. Lett. 87, 152002 (2001) [Erratum-ibid. 88, 249901 (2002)] [arXiv:hep-ph/0107158].

[24] J. W. Chen and X. D. Ji, Phys. Rev. Lett. 88, 052003 (2002) [arXiv:hep-ph/0111048].

[25] A. V. Belitsky and X. D. Ji, Phys. Lett. B 538, 289 (2002) [arXiv:hep-ph/0203276].

[26] V. Bhansali and H. Georgi, arXiv:hep-ph/9205242. 